\title{
Direct Simulation Monte Carlo Study of Orifice Flow
}

\author{
G D Danilatos \\ ESEM Research Laboratory \\ 98 Brighton Boulevarde, North Bondi, NSW 2026, Australia
}

\begin{abstract}
A study of the flow properties of argon through an orifice has been performed with the direct simulation Monte Carlo method. The study covered the full extent of the transition regime between free-molecule and continuum flow, both in the upstream and downstream regions. Results for Mach number, number density, velocity and temperature are shown for three representative cases for a specified geometry with argon gas. The variation of molecule flow rate through the orifice and the variation of mass-thickness of the gas downstream of the orifice are given in the complete transition range. The molecule flow rates computed herewith show good agreement with previously published experimental measurements. The isentropic equations of a perfect gas are shown to reproduce the expected relationship between properties as a function of the computed Mach number in the continuum regime, but they clearly deviate elsewhere, as expected. The theoretical density function and flow rate agree well with the computed values in the freemolecule flow. However, the computed flow rate is less than the flow rate of a nozzle in the continuum flow. Empirical equations for both the characteristic speed and normalized number thickness have been derived and shown to predict well the values of any gas, for all practical purposes.
\end{abstract}

\section{INTRODUCTION}

There have been extensive studies of rarefied gas flows through nozzles of various shapes but only limited information is available on flows through an orifice made on a thin plate, especially as such information relates to newly developed equipment. For example, the advent of environmental scanning electron microscopy (ESEM) requires the use of differential pumping systems for the transfer of an electron beam from a high vacuum to a high pressure environment (1). Conventionally, an orifice is used for the separation of two regions with a substantial gas pressure difference, whereby the gas flow properties both in the near and far field region of the orifice can have a significant impact on the performance of the instrument.

It is beyond the scope of this article to provide a thorough survey and fundamental gas dynamics theories related to this work. The gas densities and orifice sizes of interest correspond to the entire regime from free-molecule to continuum flow, as the high pressure region may vary from high vacuum to near atmospheric pressures while the orifice diameter is of the order of $1 \mathrm{~mm}$ or less. The available theoretical solutions are inadequate or impractical to use for certain geometries, especially in the rarefied gas flow regime. Theoretical and numerical methods $(2,3)$ as well as experimental ones $(4,5,6)$ can be used up to a certain point.

The advent of the direct simulation Monte Carlo (DSMC) method in conjunction with the availability of fast Personal Computers (7) have opened an easy and practical way to study systems, in which the variation of geometry and gas state are critical in the determination of an optimum condition of operation. The method has already been applied successfully to the study of pumping properties of an annular supersonic jet, which has led to a novel differential pumping system (8). It has also been applied to study the effect of varying the lip angle and thickness of an orifice wall, the effect of a specimen presence upstream of the orifice entrance and the effect on two combined orifices in a two stage differential pumping system. The present article is part of a series of reports dealing with such studies.

In particular, the purpose of this article is to revisit the fundamental problem of gaseous flow through an orifice with the use of the DSMC method, the results of which are compared with some existing experimental work. By establishing good agreement between computed and experimental results for some flow property, when possible, we first ensure that the computational parameters have been chosen correctly so that we may assume that the other corresponding flow properties are also correct. The property of density variation along the axis, molecular thickness 
of a gas layer and leak rate through the orifice are of direct practical application to instruments employing a charged particle beam. These properties are reported together with the Mach number, velocity, temperature and pressure of gas in the transition regime from free-molecule to near continuum flow.

\section{METHOD}

The direct simulation Monte Carlo method uses some thousands or millions of simulated molecules for the computer modeling of a real gas flow. The position and velocity of the molecules are stored and modified in the computer with time as the gas flows within given boundary conditions. The number of simulated molecules needed depends on the gas density and spatial extent of the flow field, which determine the computation time required with a given computer. In this work, we are interested in the properties of the steady state situation.

The orifice used has $0.5 \mathrm{~mm}$ diameter on a $0.1 \mathrm{~mm}$ thick plate with a diverging lip at 45 degrees angle. This geometry presents a near minimum jet mass thickness to be overcome by a charged particle beam, and has been chosen from a series of tests involving variation of plate thickness and angle. Argon is used as the test gas at given input densities $n_{0}$ between $4.94 \times 10^{20} \mathrm{mols} / \mathrm{m}^{3}$ and $1.235 \times 10^{24} \mathrm{mols} / \mathrm{m}^{3}$, which correspond to input pressures $p_{0}$ between $2 \mathrm{~Pa}$ and $5000 \mathrm{~Pa}$ at $293 \mathrm{~K}$ temperature, whilst in all cases the gas exhausts in vacuum. The similarity of flow principle has also been tested by varying the orifice diameter $D$ up to five orders of magnitude inversely to the input pressure, i.e. by keeping the product $p_{0} D$ constant; it was found that the same flow property reproduced when we normalized the field over the diameter and input property. The flow properties were studied for different values of the parameter $p_{0} D$, which has direct engineering use in ESEM technology (e.g. in detection efficiency, gain, etc.) and was preferred over the Reynolds number frequently employed in other gas dynamics problems.

The computational requirements of the DSMC method were satisfied by and large, namely, regarding the time step, cell size and minimum number of simulated molecules in each cell. However, for the very high pressure case, only the number of real molecules represented by each simulated molecule was adjusted together with the appropriate time step, while the cell size was kept the same. In the latter case, the mean free path is smaller than the cell size but this should be allowed, as there is practically no variation of the flow properties across the size of any given cell. Otherwise, the required computing time would be prohibitive at the high pressure.

The computer program yields, among other parameters, the number density, velocity, Mach number and temperature in the flow field together with the transfer rate of molecules across interfaces between zones. The transfer rate $N$ of molecules across an interface is given by

$$
N=\iint n v d A
$$

where $n$ is the number density, $v$ is the velocity and $d A$ a surface element on the interface over which we integrate. From this, we derive the leak rate and conductance that characterize the orifice, as used in vacuum technology. To generalize the applicability of results, we give the characteristic orifice speed $s_{c}$ defined by

$$
s_{c}=\frac{N}{n_{0} A}=\frac{1}{n_{0} A} \iint_{A} n v d A
$$

where we normalize the transfer rate over the input number density and the orifice area $A$. This is effectively an average velocity of the gas across the orifice at stagnation conditions, i.e. at $n_{0}$ density and $T_{0}$ temperature.

Another engineering parameter is the mass thickness or, preferably, the molecular thickness, which is the total number of gas molecules per unit area in a column of gas. The molecular thickness multiplied with the total scattering cross-section of an electron yields the average number of electron collisions, which enters as the negative exponent in the exponential decay of intensity of a focussed electron beam transferred through said column. The molecular thickness of a column along the axis stretching from the entry plane of the orifice downstream to infinity is finite, as will be shown. The latter quantity normalized over the input number density and the orifice diameter yields a dimensionless constant $\zeta_{c}$, the characteristic normalized molecular thickness, given by the definite integral

$$
\zeta_{c}=\frac{1}{n_{0} D} \int_{0}^{\infty} n d x
$$




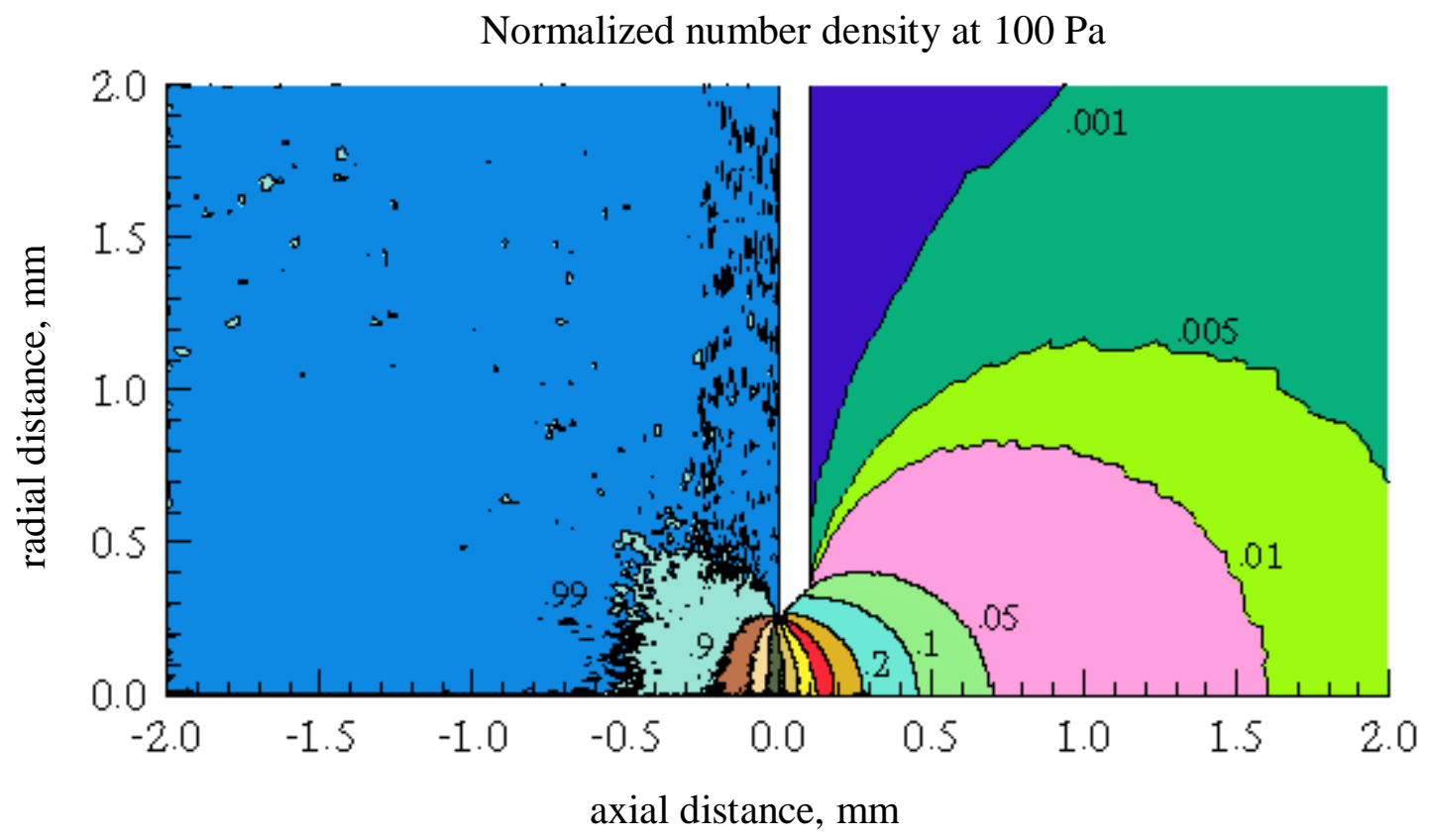

FIGURE 1. Normalized number density contours for argon supplied at $100 \mathrm{~Pa}$ (left) and exhausted in vacuum (right) through axially symmetric orifice; non-labeled contours drawn at .1 interval.

\section{RESULTS}

Some representative results are given below. The flow field shown in figure 1 displays the normalized number density contours for the case where $p_{0} D=0.05 \mathrm{~Pa}-\mathrm{m}$, e.g. for $p_{0}=100 \mathrm{~Pa}\left(\right.$ or $n_{0}=2.47 \times 10^{22} \mathrm{mols} / \mathrm{m}^{3}$ ) and $D=0.5 \mathrm{~mm}$. Because the field is axially symmetric only one half of it is shown with the axis drawn as the abscissa. The contour patterns follow the generally expected shapes for a flow through an orifice. The purpose is to establish precise quantitative results for the orifice geometry shown, such as the variation of gas density along the axis, or another direction, along which a charged particle beam may propagate. It will be seen that the onset of the transition regime, or the end of the free-molecule flow occurs around $p_{0} D=0.001 \mathrm{~Pa}-\mathrm{m}$ and is almost complete around $p_{0} D=1 \mathrm{~Pa}-\mathrm{m}$, where we enter the continuum flow.

The properties given further below are along the axis of the system at the corresponding pressures of 2,100 and $2000 \mathrm{~Pa}$. Actually, the curves are taken slightly off the axis (at $0.05 \mathrm{~mm}$ ), because there is increased scatter on the axis itself. Figure 2 shows the computed Mach number along the axis. Figure 3 shows the variation of normalized number density along the axis for the same corresponding pressures. In the latter figure, we also present values (points) of normalized density calculated with the equations of isentropic flow of a perfect gas (circles and crosses) and values (diamonds) calculated from theory in free-molecule flow, both of which are discussed later.

The velocity variation along the axis of the system is shown in figure 4 and the normalized temperature in figure 5. The corresponding isentropic flow equations of a perfect gas were also used to calculate the velocity and temperature for the three pressures using the Mach numbers of figure 2. Similar results were found, namely, there was a clear deviation at 2 and $100 \mathrm{~Pa}$ but practically no deviation at $2000 \mathrm{~Pa}$.

Figure 6 presents the characteristic speed of the orifice as we vary the pressure, or $p_{0} D$ : The solid points and line show the computed characteristic speed of the orifice as defined by equation (2). The open circles shown in the figure correspond to experimental measurements of the flow rate conducted for an orifice by Liepmann (6).

Liepmann presents his measurements as a function of Reynolds number $R e$, for which he uses as parameters the diameter of the orifice and the stagnation values of density $\rho_{0}$, viscosity $\mu_{0}$ and a velocity at the input of the gas. Specifically, as velocity he uses the formula $\left(p_{0} / \rho_{0}\right)^{1 / 2}$, which corresponds to the speed of sound divided by $\gamma^{1 / 2}$, so that the following transformation between $R e$ and $p_{0} D$ was used: 


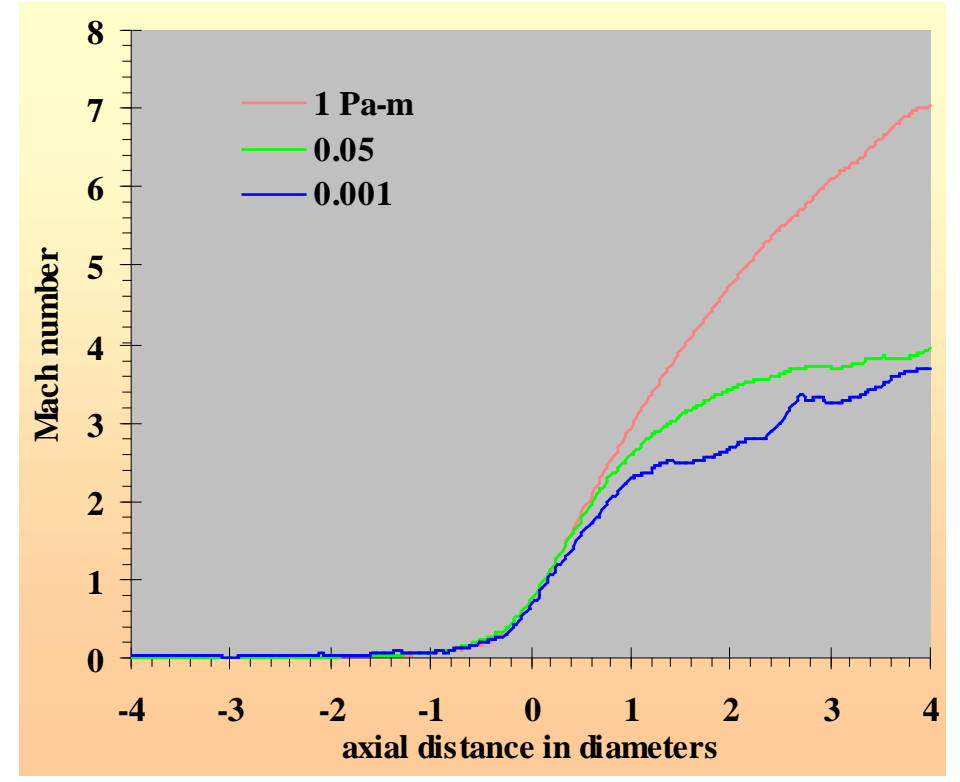

FIGURE 2. Mach number variation along orifice axis at three $p_{0} D$ values..

$$
R e=\frac{1}{\mu_{0}} \sqrt{\frac{\mathrm{M}}{R T_{0}}} p_{0} D \equiv k p_{0} D
$$

where $\mathrm{M}$ is the molecular weight of the gas and $k$ the conversion factor between the two variables. We used $\mu_{0}$ $=2.237 \times 10^{-5} \mathrm{SI}$ units, and $T_{0}=293 \mathrm{~K}$, whilst the exact values used for these, or the pressures, were not reported in the experimental work.

The characteristic normalized molecular thickness is found according to equation (3), but, in practice, it was sufficient to integrate along the axis from the entry plane of the orifice up to 20 diameters upstream in the flow, where the density is reduced to a negligible value. It is pertinent to consider also another characteristic aspect of the flow, namely, the depletion zone upstream of the orifice, where the molecular thickness is less than it would be if the gas had a homogeneous constant density (i.e. the stagnation density). The latter zone is also of interest in problems of electron beam scattering where the electron beam would suffer less losses than in a stagnation density region. By

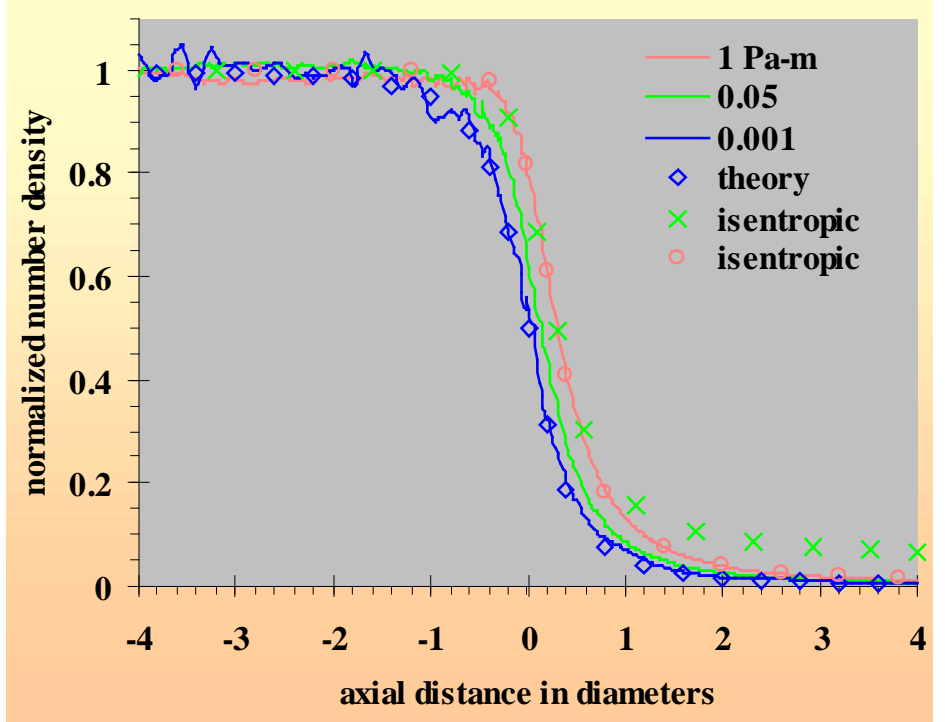

FIGURE 3. Normalized number density variation along orifice axis at three input pressures; points are calculated values by isentropic equation for $p_{0} D=1$ (circles) and $0.05 \mathrm{~Pa}-\mathrm{m}$ (crosses) and by free-molecule theory for $p_{0} D=0.001 \mathrm{~Pa}-\mathrm{m}$ (diamonds). 


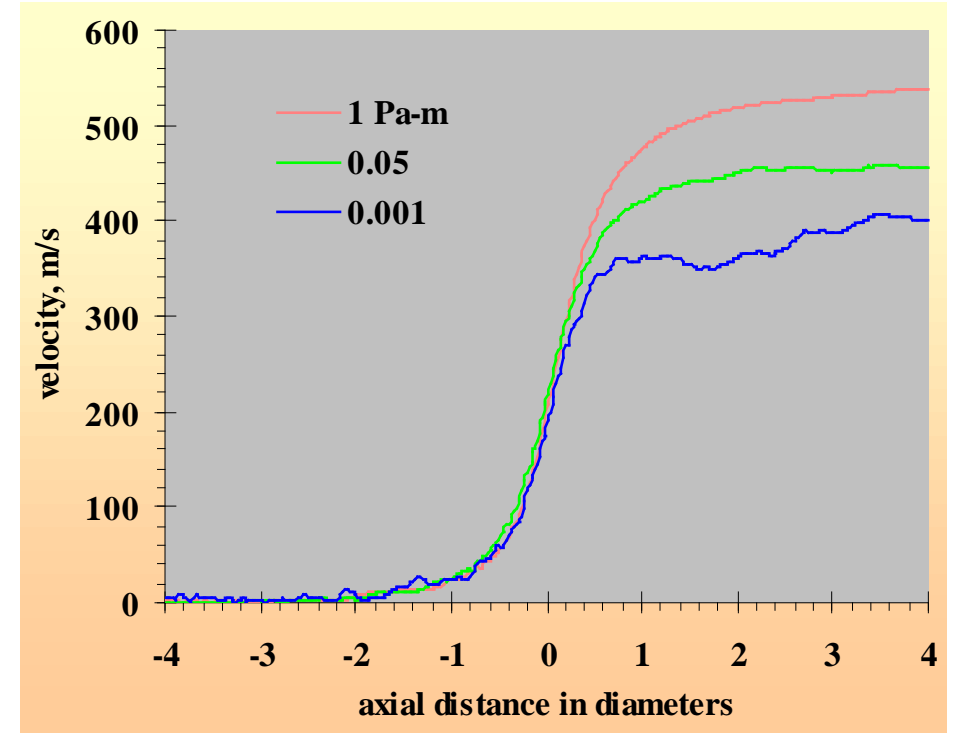

FIGURE 4. Velocity variation along orifice axis at three $p_{0} D$ values.

integrating the difference of the normalized number density from unity according to equation (3) in the negative axis, we can find the "missing", or depletion, molecular thickness. The latter subtracted from the characteristic normalized molecular thickness, results in an effective characteristic molecular thickness $\zeta_{c e}$ for the orifice. The numerical values of these properties are tabulated in table 1 and plotted in figure 7.

TABLE 1. Characteristic orifice speed $s_{c}$ and characteristic $\zeta_{c}$ and effective $\zeta_{c e}$ molecular thickness against $p_{0} D$.

\begin{tabular}{lccc}
\hline $\boldsymbol{p}_{\boldsymbol{0}} \boldsymbol{D}, \mathbf{P a}-\mathbf{m}$ & $\boldsymbol{s}_{\boldsymbol{c}}, \mathbf{m} / \mathbf{s}$ & $\zeta_{c}$ & $\zeta_{c e}$ \\
\hline 0.001 & 98.5 & 0.28 & 0.10 \\
0.005 & 102.0 & 0.29 & 0.07 \\
0.025 & 113.6 & 0.32 & 0.18 \\
0.05 & 125.6 & 0.37 & 0.28 \\
0.1 & 136.8 & 0.43 & 0.40 \\
0.25 & 144.9 & 0.50 & 0.48 \\
0.5 & 146.7 & 0.53 & 0.43 \\
1 & 146.9 & 0.53 & 0.42 \\
\hline
\end{tabular}

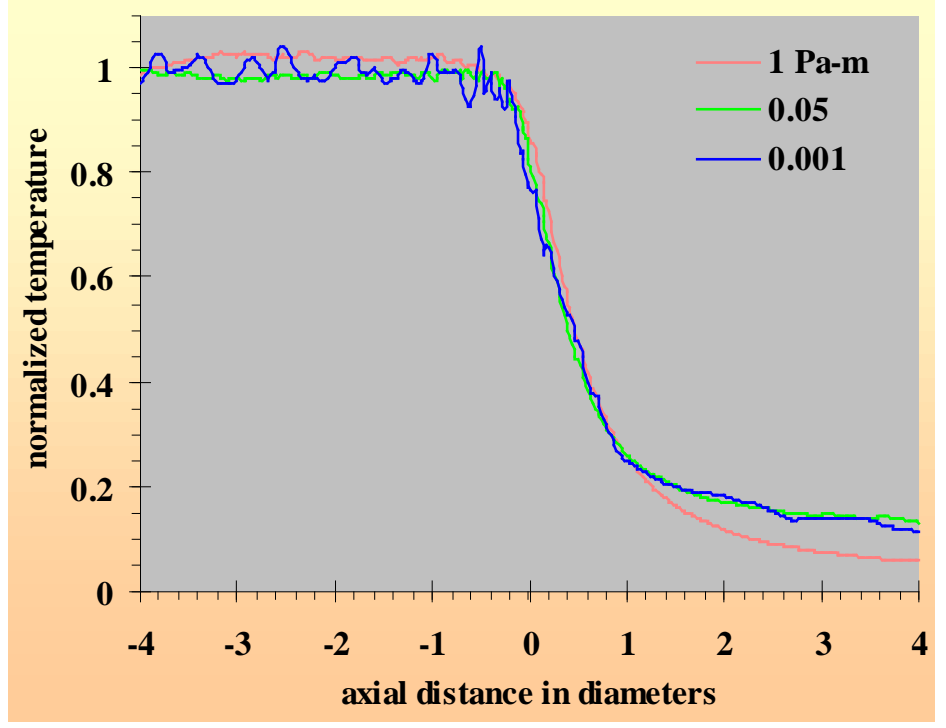

FIGURE 5. Normalized temperature variation along orifice axis at three $p_{0} D$ values. 


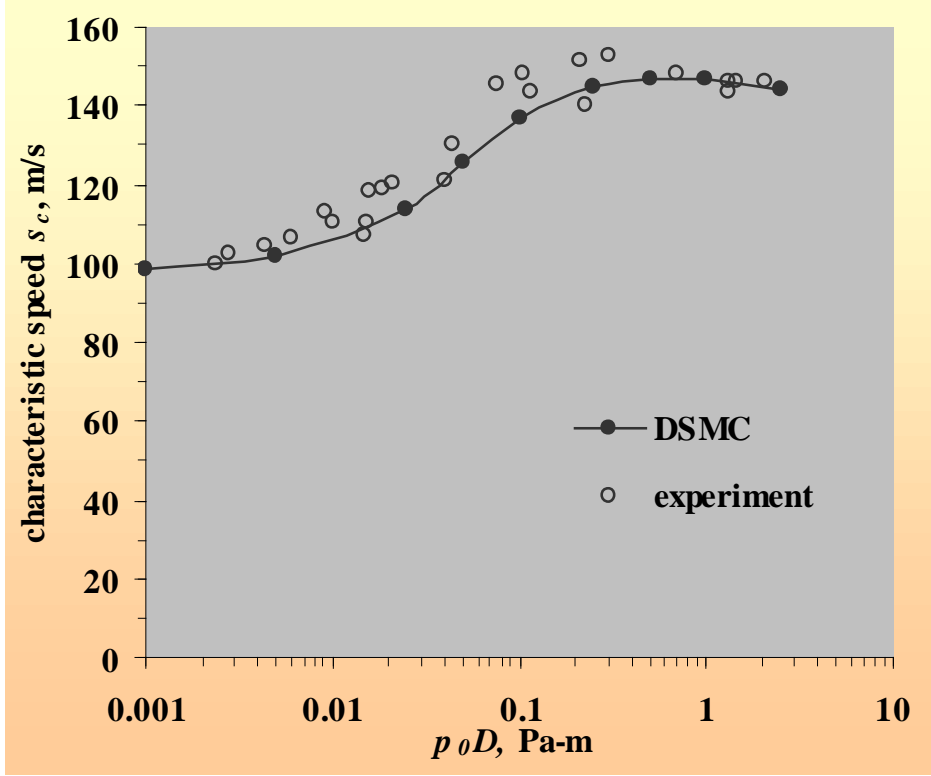

FIGURE 6. Characteristic orifice speed $s_{c}$ against $p_{0} D$ by DSMC and experiment.

\section{ANALYSIS AND DISCUSSION}

The problems presented and time required in experimental work even for an apparently simple task such as the measurement of the flow rate through an orifice (6). The DSMC method has been shown to yield quantitative understanding of all the flow properties with relative ease. This method has been particularly useful when we wish to arbitrarily vary the details of orifice geometry, pressure and gas. Analytical and other mathematical approaches to this problem are difficult even in free molecular flow (9), or impossible to apply, especially in the transition regime.

Below we compare the DSMC results with theory and derive equations for the characteristic speed and molecular thickness, which predict the behavior of other gases as well. At the high pressure end, the isentropic equations should be applicable to calculate one property from another. The variation of Mach number along the axis cannot be

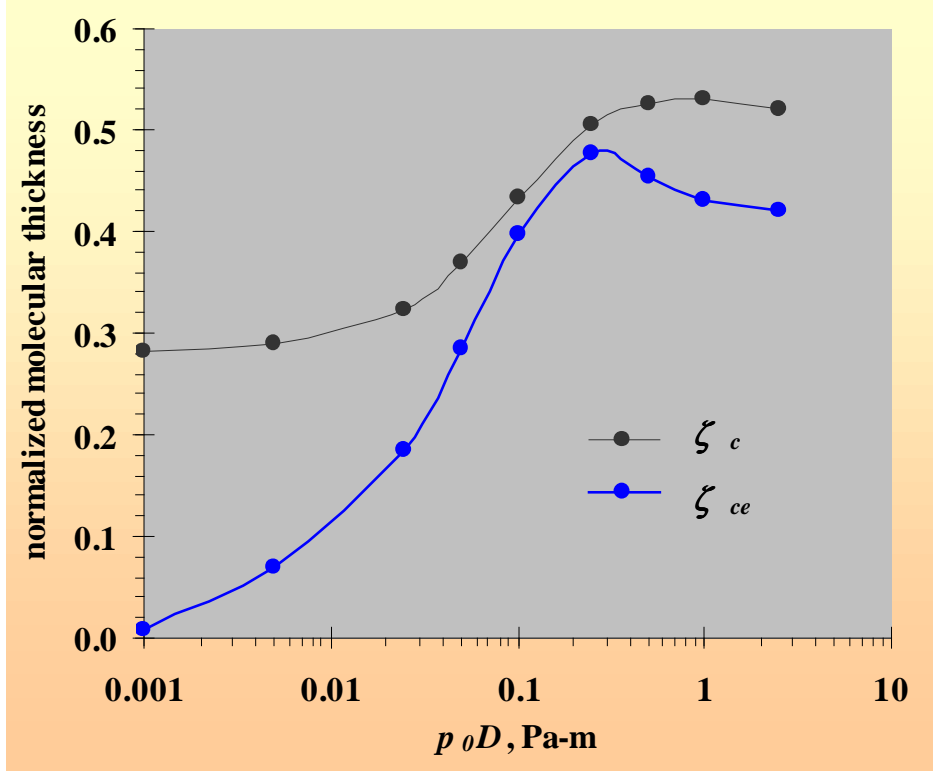

FIGURE 7. Characteristic $\zeta_{c}$ and effective $\zeta_{c e}$ normalized molecular thickness against $p_{0} D$ by DSMC. 


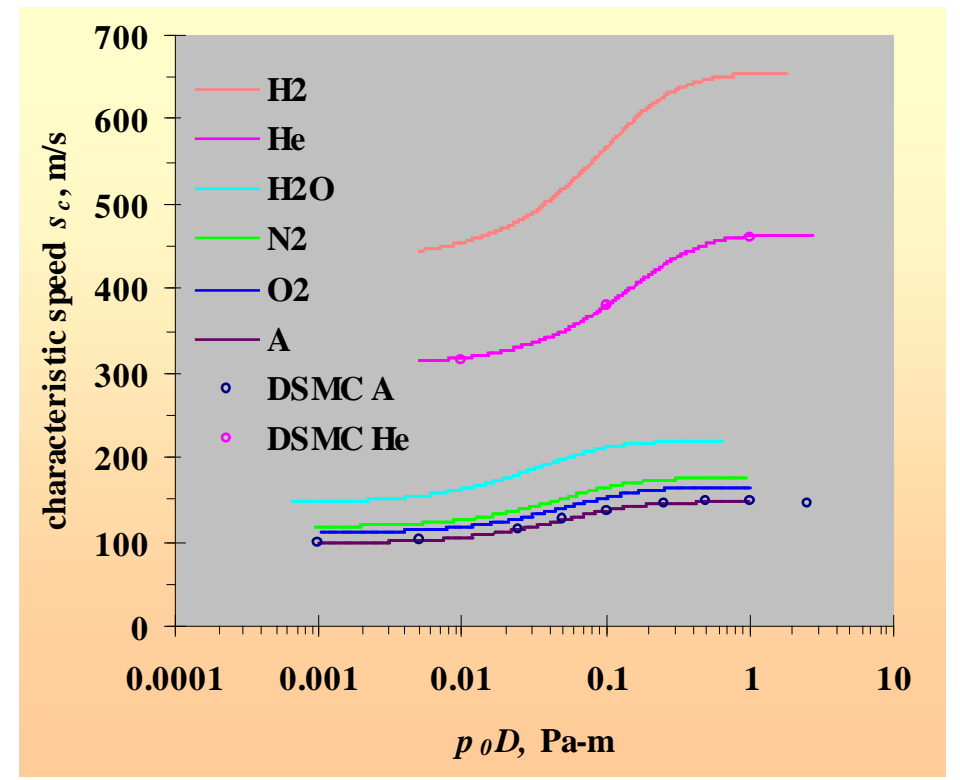

FIGURE 8. Characteristic $\mathrm{s}_{c}$ for various gases predicted (curves) and directly computed by DSMC (points).

known a priori for the given orifice, but if we use the computed values, we should predict, for example, the normalized density from the isentropic equation

$$
\frac{n}{n_{0}}=\left(1+\frac{\gamma-1}{2} M\right)^{\frac{1}{1-\gamma}}
$$

where $\gamma$ is the specific heats ratio ( $\gamma=1.667$ used for argon) and $M$ the Mach number. The points shown in figure 3 with open circles are calculated values at $2000 \mathrm{~Pa}$ and they practically coincide with the DSMC computed line at the same pressure. The points shown with $\times$ are calculated values at $100 \mathrm{~Pa}$ and they show a clear deviation from the DSMC line computed at the same pressure, which is to be expected in the transition regime. A similar deviation is found at $2 \mathrm{~Pa}$, not shown for clarity in the figure. The diamond points falling on the curve at $2 \mathrm{~Pa}$ were calculated with an equation for free-molecular flow conditions as shown further below.

The characteristic speed of an orifice under free-molecule flow is given by the equation

$$
s_{c 1}=\sqrt{\frac{R T_{0}}{2 \pi \mathrm{M}}}
$$

where $R$ is the universal gas constant and $M$ the molecular weight. From this we find for argon $s_{c l}=98.52 \mathrm{~m} / \mathrm{s}$, which is in good agreement with the lowest value in table 1. At the other extreme, the continuum flow theory gives,

$$
s_{c 2}=\sqrt{\frac{\not R T_{0}}{\mathrm{M}}\left(\frac{2}{\gamma+1}\right)^{\frac{\gamma+1}{\gamma-1}}}
$$

from which we find $s_{c 2}=179.34 \mathrm{~m} / \mathrm{s}$. The latter value is clearly above the highest value of $146.9 \mathrm{~m} / \mathrm{s}$ in table 1 . The shape of speed curve in figure 6 indicates that we have reached the continuum flow regime. Equation (7) is applicable to an ideal nozzle situation and the difference may be attributed to the different geometry of the orifice and the real gas simulation of the flow including boundary layer and vena contracta effects. It is interesting that the approach to continuum flow shows a slight overshoot on the speed curve in both the experiment and DSMC. In future work, a repeat of the computations at the very highest pressure and beyond with much smaller cell size may be necessary to establish the veracity of this feature.

Liepmann measured the flow rate for different gases and found that the normalized value plotted against Reynolds number is independent of the gas nature. The normalization was effected by dividing by the limiting value of flow rate in the free-molecule regime corresponding to equation (6). We can apply and test this finding by using the present results. If the DSMC computed points in figure 6 are fitted with a function

and transform variables by

$$
s_{c}^{\prime}=\mathrm{f}\left(p_{0}^{\prime} D^{\prime}\right) \text { with } 0.001 \leq p_{0}^{\prime} D^{\prime} \leq 1
$$




$$
s_{c}^{\prime}=\frac{s_{c 1}^{\prime}}{s_{c 1}} s_{c} \text { and } p_{0}^{\prime} D^{\prime}=\frac{k^{\prime}}{k} p_{0} D
$$

we obtain the equivalent function for any other gas

$$
s_{c}=\frac{s_{c 1}}{s_{c 1}^{\prime}} \mathrm{f}\left(\frac{k^{\prime}}{k} p_{0} D\right) \text { with } 0.001 \frac{k}{k^{\prime}} \leq p_{0} D \leq \frac{k}{k^{\prime}}
$$

where the primes refer to argon and the constants are taken from equations (4) and (6). One may use any satisfactorily fitting curve in equation (8), but to illustrate the consequences and provide a ready solution, we have used the fitted curve

$$
y=\frac{1+b x+d x^{2}}{a+c x+e x^{2}}
$$

where the constants $a, b, c, \mathrm{~d}$ and $e$ are given in table 2 . The resulting function of characteristic speed is plotted in figure 8 for several gases.

Three additional points were then computed for helium and, when plotted in the same figure, they were found to practically fall on the predicted curve for helium. One more point for a diatomic gas, namely, nitrogen was computed and also found to fall on its corresponding curve, but not shown in the figure for clarity. Therefore, both Liepmann's measurements and the DSMC method corroborate each other and show that we can have a universal equation to predict the speed for other gases.

The normalized number density shows a similar trend as the flow rate. One wonders if there is also a transformation to predict the behavior of this parameter for other gases. Whereas we can expect the transformation of $p_{0} D$ to be the same, the corresponding one for $\zeta_{c}$ is not initially obvious and we do not have any experimental measurements. The DSMC computation for helium showed that the $\zeta_{c l}$ value is the same as for argon. The latter finding is reasonable by considering that, while helium has higher speed through the orifice, the jet downstream is proportionally more dilute, so that the total number of particles could be the same, if we start from the same stagnation number density in a free-molecule flow. The constancy of $\zeta_{c l}$ for all gases is a consequence of Avogadro's law. This leads us to use a corresponding function $\mathrm{f}\left(p_{0} D\right)$ of the computed values of molecular thickness for argon and deduce a relationship

$$
\zeta_{c}=\mathrm{f}\left(\frac{k^{\prime}}{k} p_{0} D\right) \text { with } 0.001 \frac{k}{k^{\prime}} \leq p_{0} D \leq \frac{k}{k^{\prime}}
$$

for any other gas, by transforming only the $p_{0} D$ variable. To test this hypothesis, we fitted the argon points with a function of the same form as in equation (11), but with a new set of constants $a, b, c, \mathrm{~d}$ and $e$ also given in table 2 . The results are plotted in figure 9 for the same gases. The computed values for helium are also plotted and they seem to practically fall on the predicted curve. The same success was obtained for the nitrogen point, not shown in the figure for clarity.

TABLE 2. Constants used in equation (11) for $s_{c}$ and $\zeta_{c}$

\begin{tabular}{lccccc}
\hline \multicolumn{1}{c}{$\boldsymbol{a}$} & $\boldsymbol{b}$ & $\boldsymbol{c}$ & $\boldsymbol{d}$ & $\boldsymbol{e}$ \\
\hline $\boldsymbol{s}_{\boldsymbol{c}}$ & 0.010223485 & 21.50653875 & 0.138615757 & 206.0043412 & 1.406711342 \\
$\boldsymbol{\zeta}_{\boldsymbol{c}}$ & 3.570661537 & 12.15808873 & 19.574990410 & 95.48530701 & 181.7056835 \\
\hline
\end{tabular}

Since we have stumbled on a universal constant for the molecular thickness in the free-molecule flow, it is thought to theoretically derive this constant for a thin orifice, for which the number density function can be easily derived and integrated over distance. The number density function along the axis on the vacuum side of the orifice is simply proportional to the solid angle subtended by the orifice area at any point. By dividing the solid angle by $4 \pi$ sterads and the distance by $D$, we get the normalized number density vs. normalized distance as

$$
\frac{n}{n_{0}}=\frac{1}{2}-\frac{z}{2 \sqrt{z^{2}+0.25}}
$$

It can be easily shown that the same function gives also the density for negative $z$ (in the upstream region), where the shape of the function is symmetrical with respect to the center of the orifice. First, we plot a number of points calculated by equation (13) in figure 3 (diamonds) and observe that they practically fall on the DSMC computed curve for $2 \mathrm{~Pa}$, as it should. Second, by integrating the latter function according to equation (3), we find the universal dimensionless constant of 0.25 . A corresponding constant for the "missing" particles in the depletion region can be found by integrating the difference from unity. The latter is also found to be 0.25 , which is to be expected for an extremely dilute gas. As the pressure increases, even in the free-molecule flow (defined with respect 


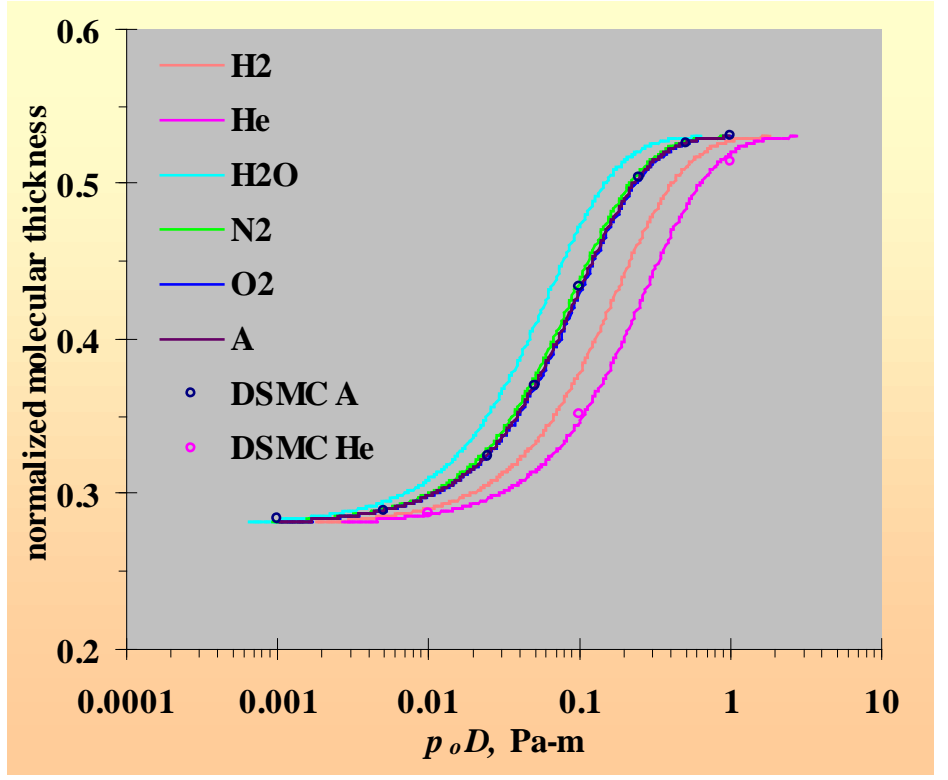

FIGURE 9. Characteristic $\zeta_{c}$ for various gases predicted (curves) and directly computed by DSMC (points).

to the diameter of the orifice) the density function becomes skewed with higher density towards the vacuum side, so that the effective number density is greater than zero. The results shown in figure 7 are generally consistent with this analysis. The $\zeta_{c e}$ seems to practically approach the value of $\zeta_{c}$ as we approach continuum flow and then it deviates a little again. The latter deviation is due to the slightly lower computed values of the number density in the depletion zone over a more extensive range upstream. It is correspondingly found that the temperature increases slightly in the depletion zone in such a way that the contours of pressure show a variation only very close to the orifice and is equal to the stagnation value everywhere else in the upstream region. The inverse variation of number density and temperature at constant pressure on the upstream side of the orifice has also been observed, but at the lower input pressure as can be seen in figure 5 .

The precise nature of these phenomena is not clear at this stage. It may be a relatively long-term variation of temperature effects, which would disappear if we computed for much longer time, or it may be some oscillation of low frequency. These phenomena, considered "weak" for our practical purposes, may also relate to a small overshoot observed on the curves of speed and number thickness. However, for all practical purposes, the $s_{c}$ and $\zeta_{c}$ are sufficient to quantitatively describe the performance of an orifice. It should be pointed out that bellow $p_{0} D<0.1$ $\mathrm{Pa}-\mathrm{m}$, where $\zeta_{c e}$ differs significantly from $\zeta_{c}$, the number density in absolute terms is so low that an electron beam suffers practically no or very little scattering. The $\zeta_{c}$ is the dominant parameter for $p_{0} D>0.1 \mathrm{~Pa}-\mathrm{m}$, even more so for other geometry (e.g. cylindrical), so that this parameter alone can be used as a measure of performance in aperture design.

The "weak" phenomena can become the subject of more refined computations and experimental work in the future. In the meantime, some additional computations have already been performed to establish the magnitude of possible errors. It has been found that any errors present seem to arise mainly from the way the DSMC program is applied rather than by the program itself. With cell size, time step and number of simulated molecules being set correctly, the user is left to choose a satisfactory time interval for sampling the steady-state situation and a satisfactory boundary condition especially for the input gas. The orifice flow seems to approach the steady-state condition within a few percent in two to four days of computation time with a Pentium-II/266 and a Pentium-II/450 type of PC used for this work. The dilute gas case at $p_{0} D=0.001 \mathrm{~Pa}-\mathrm{m}$ was allowed to run up to two weeks. Even at this low pressure, we still need a large number of simulated molecules, or very long computation time to reduce the statistical variation to an acceptable level. We also need a large number of small cells in the immediate neighborhood of the orifice, where the flow properties have a sharp gradient. By this investigation, we found that that the $s_{c l}$ was about $1.5 \%$ less than the initial value, of which the coincidence with the theoretical value must have been fortuitous. This difference seemed to be due to the small fraction of molecules back-scattered from the lip surface of the orifice. To further test this, the orifice lip thickness was set to zero and the input boundary placed at the orifice plane. The steady-state was attained at much shorter times and the equilibrium value was found indeed to be $98.5 \mathrm{~m} / \mathrm{s}$, as it should, because the theoretical formula assumes that the rate through the orifice is the same as the 
rate at which particles strike a surface at stagnation conditions. Then the boundary was shifted to $0.5,1,2,4$ and 8 diameters upstream, and found the same constant value of $98.5 \mathrm{~m} / \mathrm{s}$. It should be noted that the stagnation mean free path is around 6 diameters and, therefore, the input boundary is the determinant factor (supply) for the orifice flow rate. The corresponding $\zeta_{c}$ is a little above 0.25 and the $\zeta_{c e}$ is a little above zero, as predicted by theory.

The initial state of gas set inside the flow field in a DSMC test also affects the direction from which the steadystate condition is approached and hence the sign of any residual small error. If the initial state in the downstream region has a higher density than the equilibrium value, the molecular thickness can be slightly overestimated, whilst the reverse happens if we start from vacuum. In the upstream region, significant perturbations occur if we start from vacuum, while a "smooth" approach takes place if we start from stagnation condition.

A last test was performed by setting the input boundary at 20 diameters upstream of the original orifice having $0.1 \mathrm{~mm}$ thickness at 45 degrees lip angle. This showed that both the orifice and its lip perturbed the flow in a way that resulted in further but small reduction of flow rate and molecular thickness, as well as in a redistribution of the depletion zone and density contours close to the stagnation value in the upstream region. The distribution of the back-scattered fraction of particles has been described for cylindrical geometry by Dayton (9) and seems to be corroborated by DSMC if it is given sufficient time to resolve the phenomenon. The $\zeta_{c e}$ took on small but negative values.

The computed values in figure 6 coincide with the lower experimental points. This can be attributed either to the thicker lip used here than in the experiment, or to a small systematic error in the computed or the experimental values which show also considerable scatter. The sources of possible error were quite significant in the experiment.

However, all these phenomena have no practical significance for the purposes originally set for this work and they have not been pursued further. They can have important theoretical and practical value in other areas of research and the DSMC method seems the only way to access them and study them in considerable detail with but higher capacity computers.

\section{CONCLUSION}

The DSMC method can be applied with confidence in a variety of gas flow regimes, especially where other methods are difficult, impractical or impossible to use. A study of the flow properties of argon through an orifice has covered the full extent of the transition regime between free-molecule and continuum flow for a specified geometry. Results for Mach number, number density, velocity and temperature are shown for three representative cases. The equations of isentropic flow for a perfect gas reproduce the interrelationship among computed values as expected at high pressure, and the low pressure computations are confirmed by theory. The variation of molecule flow rate through the orifice and the variation of molecular thickness of the gas downstream the orifice are shown in the complete transition range. The flow rates computed herewith show good agreement with previously published experimental measurements. Empirical equations are derived to predict the speed and molecular thickness for any gas and this has been confirmed with additional DSMC computations for helium and nitrogen. The orifice gas flow can now be quantified and understood. Quantitative knowledge of these and other flow properties is indispensable for an optimum instrument design. The advent of the DSMC method has given a new impetus in many areas, especially where advanced gas dynamics expertise was not readily available.

\section{ACKNOWLEDGMENT}

Professor GA Bird is gratefully acknowledged for providing the DSMC computational programs and for discussing important improvements of the method.

\section{REFERENCES}

1. Danilatos, G., D., Adv. Electronics and Electron Phys. 71, 109-250 (1988).

2. Beylich, A., E., Z. Flugwiss. Weltraumforsch. 3, Heft 1, 48-58 (1979).

3. Beylich, A., E., "Plane Flow Through an Orifice" in Rarefied Gas Dynamics-1984, edited by Hakuro Oguchi, Proceedings of the $14^{\text {th }}$ International Symposium on Rarefied Gas Dynamics, University of Tokyo Press, pp. 517-524.

4 Anderson, J., B., Andres, R., P., and Fenn, J., B., Adv. Chem. Phys. 10, 275-317. 
5. Ashkenas, H., and Sherman, S., F., "Experimental Methods in Rarefied Gas Dynamics" in Rarefied Gas Dynamics-1966, edited by JH de Leeuw, Proceedings of the $4^{\text {th }}$ International Symposium on Rarefied Gas Dynamics, Academic Press, New York, Vol. II, pp. 84-105.

6. Liepmann, H., W., J. Fluid Mech. 10, 65-79 (1961).

7. Bird, G., A., Molecular Gas Dynamics and the Direct Simulation of Gas Flows, Clarendon Press, Oxford, 1995, pp. 218-388.

8. Danilatos, G., D., Microscopy and Microanalysis 6, 21-30 (2000).

9. Dayton, B., B., Committee on Vacuum Techniques, 1956 National Symposium on Vacuum Technology Transactions, edited by Perry E. S. and Darant J. H., Pergamon Press 1957, pp. 5-11. 